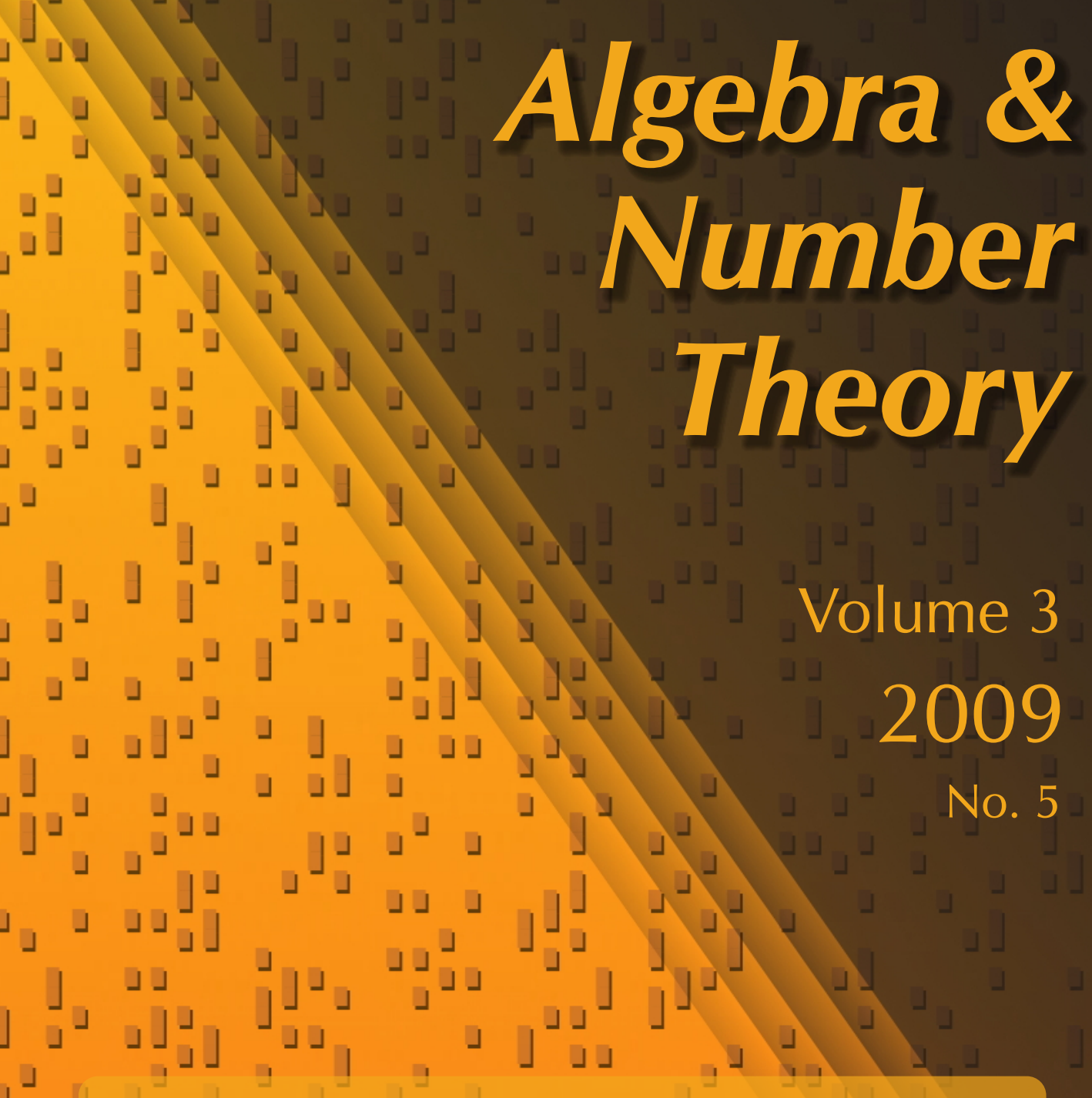

Vanishing of trace forms in low characteristics

Skip Garibaldi

Appendix by Alexander Premet

\lrcorner$\lrcorner$

」」

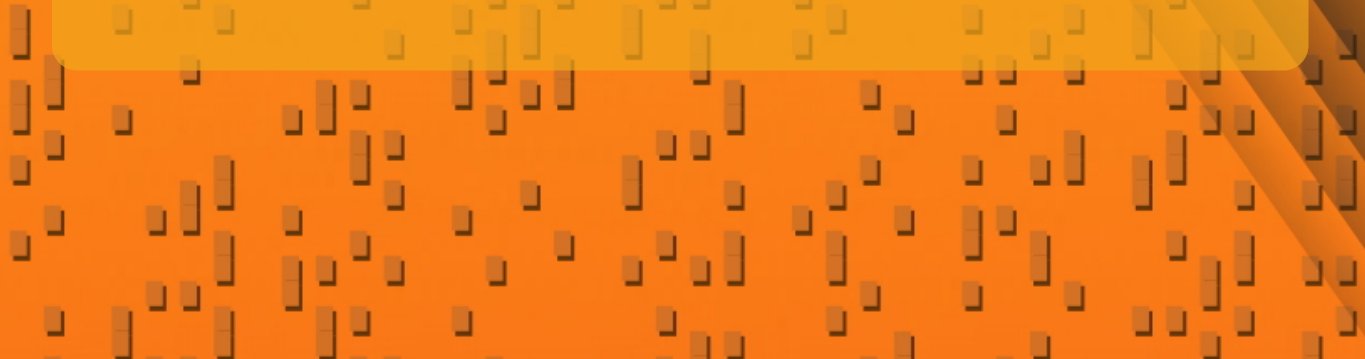

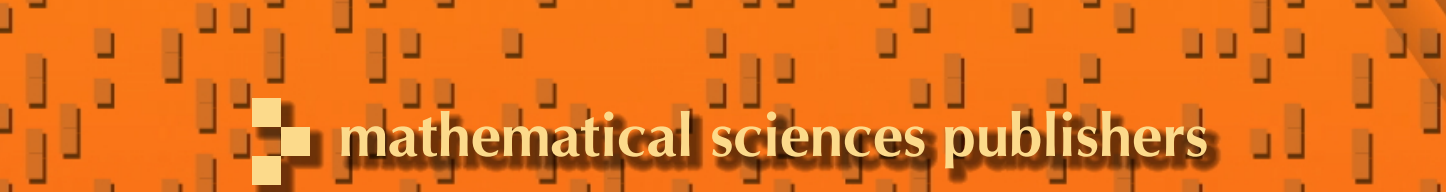




\title{
Vanishing of trace forms in low characteristics
}

\author{
Skip Garibaldi \\ Appendix by Alexander Premet
}

\begin{abstract}
Every finite-dimensional representation of an algebraic group $G$ gives a trace symmetric bilinear form on the Lie algebra of $G$. We give criteria in terms of root system data for the existence of a representation such that this form is nonzero or nondegenerate. As a corollary, we show that a Lie algebra of type $E_{8}$ over a field of characteristic 5 does not have a "quotient trace form", answering a question posed in the 1960 s.
\end{abstract}

Let $G$ be an algebraic group over a field $F$, acting on a finite-dimensional vector space $V$ via a homomorphism $\rho: G \rightarrow \operatorname{GL}(V)$. The differential $\mathrm{d} \rho$ of $\rho$ maps the Lie algebra $\operatorname{Lie}(G)$ of $G$ into $\mathfrak{g l}(V)$, and we put $\operatorname{Tr}_{\rho}$ for the symmetric bilinear form

$$
\operatorname{Tr}_{\rho}(x, y):=\operatorname{trace}(\mathrm{d} \rho(x) \mathrm{d} \rho(y)) \quad \text { for } x, y \in \operatorname{Lie}(G) .
$$

We call $\operatorname{Tr}_{\rho}$ a trace form of $G$. Such forms appear, for example, in the hypotheses for the Jacobson-Morozov Theorem [Carter 1985, 5.3.1], in Richardson's proof that there are finitely many conjugacy classes of nilpotent elements in the Lie algebra of a semisimple algebraic group as in [Jantzen 2004, §2] or [Humphreys $1995, \S \S 3.8,3.9]$, and in the "explicit" construction of a Springer isomorphism in [Bardsley and Richardson 1985, §9.3]. We prove:

Theorem A. Let $G$ be a split and almost simple linear algebraic group over a field $F$.

(1) There is a representation $\rho$ of $G$ with $\operatorname{Tr}_{\rho}$ nondegenerate if and only if the characteristic of $F$ is very good for $G$.

(2) There is a representation $\rho$ of $G$ with $\operatorname{Tr}_{\rho}$ nonzero if and only if the characteristic of $F$ is as indicated in Table I.

A weaker version (up to isogeny) of the "if" direction of part (1) is standard; see for example [Springer and Steinberg 1970, I.5.3] or [Carter 1985, 1.16]. (After this paper was released as a preprint, I learned that Alexander Premet had previously

MSC2000: primary 20G05; secondary 17B50, 17B25.

Keywords: trace form, E8, Richardson's condition, Dynkin index. 


\begin{tabular}{|c|c|c|}
\hline$G$ & $\begin{array}{c}\text { Every } \rho \text { has } \operatorname{Tr}_{\rho} \\
\text { degenerate if char } F\end{array}$ & $\begin{array}{l}\text { Every } \rho \text { has } \operatorname{Tr}_{\rho} \\
\text { zero if char } F\end{array}$ \\
\hline $\mathrm{SL}_{n} / \boldsymbol{\mu}_{m}$ with $m$ odd & divides $n$ & divides $\operatorname{gcd}(m, n / m)$ \\
\hline $\mathrm{SL}_{n} / \boldsymbol{\mu}_{m}$ with $m$ even & divides $n$ & divides $2 \operatorname{gcd}(m, n / m)$ \\
\hline $\mathrm{Sp}_{2 n}$ & equals 2 & [does not occur] \\
\hline $\left.\begin{array}{c}\mathrm{SO}_{n}, \mathrm{Spin}_{n}, \mathrm{PSO}_{n} \text { with } n \geq 7, \\
\mathrm{HSpin}_{4 n} \text { for } n \geq 3, \mathrm{PSp}_{2 n}\end{array}\right\}$ & equals 2 & equals 2 \\
\hline$E_{6}$ adjoint, $G_{2}$ & equals 2 or 3 & equals 2 \\
\hline $\begin{array}{c}E_{6} \text { simply connected, } E_{7}, F_{4} \\
E_{8}\end{array}$ & $\begin{array}{l}\text { equals } 2 \text { or } 3 \\
\text { equals } 2,3 \text {, or } 5\end{array}$ & $\begin{array}{l}\text { equals } 2 \text { or } 3 \\
\text { equals } 2,3 \text {, or } 5\end{array}$ \\
\hline
\end{tabular}

Table I. Primes where $\operatorname{Tr}_{\rho}$ is degenerate or zero for every $\rho$. The middle column lists the primes that are not very good for $G$. For simply connected $G$, the right column lists the torsion primes for $G$ as defined in, for example, [Steinberg 1975, 1.13].

proved the "only if" direction of part (1) for groups not of type $A_{n}$, but his proof has not been published [Premet 1995, p. 80].)

Both directions of part (1) are deduced from part (2). The crux of the proof of part (2) is a formula for the trace form $\operatorname{Tr}_{\rho}$, given in Proposition 4.1 below.

We remark that the characteristics in part (1) of the theorem depend only on the isogeny class of $G$, whereas the characteristics in part (2) of the theorem are more sensitive. For example, $\mathrm{Sp}_{2 n}$ has a representation with nonzero trace form over every field, whereas its quotient $\mathrm{PSp}_{2 n}$ has such only in characteristic different from 2; this is no surprise because $\mathrm{PSp}_{2 n}$ has fewer representations than $\mathrm{Sp}_{2 n}$. But the opposite phenomenon also occurs: a simply connected group of type $E_{6}$ has a representation with nonzero trace form only in characteristics $\neq 2,3$, whereas its quotient the adjoint $E_{6}$ has one over every field of characteristic $\neq 2$. This opposite phenomenon is related to the number $E(G)$ defined in Section 3 below.

For $G$ of type $E_{8}$, we can strengthen Theorem A. Given a representation $\psi$ of the Lie algebra $\operatorname{Lie}(G)$ of $G$, one can define a trace form $\operatorname{Tr}_{\psi}$ on $\operatorname{Lie}(G)$ by setting $(x, y) \mapsto \operatorname{trace}(\psi(x) \psi(y))$. We prove

Theorem B. If $F$ has characteristic 2, 3, or 5 and $G$ is of type $E_{8}$, then the trace form of every representation of $\operatorname{Lie}(G)$ is zero.

This is a strengthening of Theorem A because many representations $\psi$ of $\operatorname{Lie}(G)$ are not differentials of representations of $G$. The proof of Theorem B is given in 11.3; it amounts to a combination of Theorem A and a result generously provided by Premet, presented in the Appendix. The converse of Theorem B is of course true; in characteristic $\neq 2,3,5$, the Killing form is nondegenerate. 
In characteristic 5, Theorem B easily gives an apparently stronger statement, namely that $\operatorname{Lie}(G)$ has no quotient trace form, see Corollary 11.4. This answers a question posed in the early 1960s, see for example [Block 1962, p. 554], [Block and Zassenhaus 1964, p. 543], or [Seligman 1967, p. 48].

From the point of view of Lie algebras, this paper addresses the existence of restricted representations with nonzero or nondegenerate trace forms on Lie algebras of almost simple algebraic groups. These algebras are approximately the simple Lie algebras of classical type. For fields of characteristic $\geq 5$ and simple Lie algebras of other types (necessarily Cartan or Melikian by Block-Premet-StradeWilson, see [Strade 2004] or [Mathieu 2000], every representation has zero trace form by Block [1962, Corollary 3.1].

Notation. All algebraic groups discussed here are linear. Such a group $G$ over a field $F$ is almost simple if it is semisimple and has no proper connected, closed, normal subgroups defined over $F$. In case $F$ is separably closed, the almost simple algebraic groups are the semisimple groups whose Dynkin diagrams are connected.

$\mathrm{PSO}_{n}$ denotes the adjoint group of the (split) special orthogonal group $\mathrm{SO}_{n}$; when $n$ is odd it is the same as $\mathrm{SO}_{n}$. Similarly, $\mathrm{PSp}_{2 n}$ is the adjoint group of type $C_{n}$; it can be viewed as $\mathrm{Sp}_{2 n} / \boldsymbol{\mu}_{2}$. The groups $\mathrm{SO}_{n}, \mathrm{Spin}_{n}$, and $\mathrm{PSO}_{n}$ for $n=3,5$, and 6 are isogenous to $\mathrm{SL}_{2}, \mathrm{Sp}_{4}$, or $\mathrm{SL}_{4}$ and appear in Table $\mathrm{I}$ in that alternative form. For $n \geq 3$, we write $\mathrm{HSpin}_{4 n}$ for the nontrivial quotient of $\operatorname{Spin}_{4 n}$ that is neither $\mathrm{SO}_{4 n}$ nor adjoint.

\section{The number $N(G)$ and the Dynkin index}

1.1. Fix a simple root system $R$. We write $P$ for its weight lattice and $\langle$,$\rangle for$ the canonical pairing between $P$ and its dual. Fix a long root $\alpha \in R$ and write $\alpha^{\vee}$ for the associated coroot. For each subset $X$ of $P$ that is invariant under the Weyl group, we put

$$
N(X):=\frac{1}{2} \sum_{x \in X}\left\langle x, \alpha^{\vee}\right\rangle^{2} \in \mathbb{Z}\left[\frac{1}{2}\right] .
$$

The number $N(X)$ does not depend on the choice of $\alpha$ because the long roots are conjugate under the Weyl group.

Furthermore, $N(X)$ is an integer. To see this, note that the reflection $s$ in the hyperplane orthogonal to $\alpha$ satisfies $\left\langle s x, \alpha^{\vee}\right\rangle=\left\langle x, s \alpha^{\vee}\right\rangle=-\left\langle x, \alpha^{\vee}\right\rangle$, so in the definition of $N(X)$, the sum can be taken to run over those $x$ satisfying $x \neq s x$. For such $x$, we have $\left\langle x, \alpha^{\vee}\right\rangle^{2}+\left\langle s x, \alpha^{\vee}\right\rangle^{2}=2\left\langle x, \alpha^{\vee}\right\rangle^{2}$, proving the claim.

Example 1.2. The computations in [Springer and Steinberg 1970, pp. 180, 181] show that $N(R)=2 h^{\vee}$, where $h^{\vee}$ denotes the dual Coxeter number of $R$, which is defined as follows. Fix a set of simple roots $\Delta$ of $R$. Write $\tilde{\alpha}$ for the highest root; 
the corresponding coroot $\tilde{\alpha}^{\vee}$ is

$$
\tilde{\alpha}^{\vee}=\sum_{\delta \in \Delta} m_{\delta}^{\vee} \delta^{\vee}
$$

for some natural numbers $m_{\delta}^{\vee}$. The dual Coxeter number $h^{\vee}$ is defined by

$$
h^{\vee}:=1+\sum m_{\delta}^{\vee}
$$

In case all the roots of $R$ have the same length, it is the (usual) Coxeter number $h$ and is given in the tables in [Bourbaki 2002].

Suppose that there are two different root lengths in $R$; we write $L$ for the set of long roots and $S$ for the set of short roots. The arguments in [Springer and Steinberg 1970] are easily adapted to show that

$$
N(L)=2\left(1+\sum_{\delta \in \Delta \cap L} m_{\delta}^{\vee}\right) \text { and } N(S)=2 \sum_{\delta \in \Delta \cap S} m_{\delta}^{\vee} .
$$

We obtain the following numbers:

\begin{tabular}{|c|cccc|}
\hline type of $R$ & $h$ & $h^{\vee}$ & $N(L)$ & $N(S)$ \\
\hline$B_{n}(n \geq 2)$ & $2 n$ & $2 n-1$ & $4(n-1)$ & 2 \\
$C_{n}(n \geq 2)$ & $2 n$ & $n+1$ & 4 & $2(n-1)$ \\
$G_{2}$ & 6 & 4 & 6 & 2 \\
$F_{4}$ & 12 & 9 & 12 & 6 \\
\hline
\end{tabular}

Definition 1.3. Fix a split almost simple linear algebraic group $G$ over $F$. Fix also a pinning of $G$ with respect to some maximal torus $T$; this includes a root system $R$ and a set of simple roots $\Delta$ of $G$ with respect to $T$. For a representation $\rho$ of $G$ over $F$, one defines

$$
N(\rho):=\sum_{\substack{\text { dominant } \\
\text { weights } \lambda}}\left(\begin{array}{l}
\text { multiplicity of } \lambda \\
\text { as a weight of } \rho
\end{array}\right) \cdot N(W \lambda) \in \mathbb{Z} .
$$

For example, the adjoint representation Ad has $N(\mathrm{Ad})=2 h^{\vee}$ by Example 1.2. The number $N(\rho)$ is the Dynkin index of the representation $\rho$ defined in [Dynkin 1952, p. 130] and studied in [Merkurjev 2003]. The Dynkin index of the fundamental irreducible representations of $G$ (over $\mathbb{C}$ ) are listed in [Laszlo and Sorger 1997, Proposition 2.6] or [McKay et al. 1990, pp. 36-44], correcting some small errors in Dynkin's calculations.

We put

$$
N(G):=\operatorname{gcd} N(\rho),
$$


where the gcd runs over the representations of $G$ defined over $F$. Because the map $\rho \mapsto N(\rho)$ depends only on the weights of $\rho$ with multiplicity, it is compatible with short exact sequences

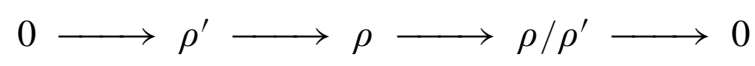

in the sense that

$$
N(\rho)=N\left(\rho^{\prime}\right)+N\left(\rho / \rho^{\prime}\right) .
$$

Writing $R G$ for the representation ring of $G$, we obtain a homomorphism of abelian groups $N: R G \rightarrow \mathbb{Z}$ with image $N(G) \cdot \mathbb{Z}$.

In the definition of $N(G)$, it suffices to let the gcd run over generators of $R G$, for example, the irreducible representations of $G$. For an irreducible representation $\rho$, the highest weight $\lambda$ has multiplicity 1 and all the other weights of $\rho$ are lower in the partial ordering. Inducting on the partial ordering, we find

$$
N(G)=\operatorname{gcd}\left\{N(W \lambda) \mid \lambda \in T^{*}\right\} .
$$

In particular, $N(G)$ depends only on the root system $R$ and the lattice $T^{*}$, and not on the field $F$.

Example 1.6. When $G$ is simply connected, the number $N(G)$ is known as the Dynkin index of $G$ and its value is listed in [Laszlo and Sorger 1997], for example. Examining the list of values, one finds that the primes dividing $N(G)$ (for $G$ simply connected) are the torsion primes of $G$.

Example 1.7. Write $\mathrm{Spin}_{n}$ and $\mathrm{SO}_{n}$ for the spin and special orthogonal groups of an $n$-dimensional nondegenerate quadratic form of maximal Witt index. For $n \geq 7$, these groups are split and almost simple of type $B_{l}$ (with $l \geq 3$ ) or $D_{l}$ (with $l \geq 4$ ). The Dynkin index $N\left(\operatorname{Spin}_{n}\right)$ is 2; it obviously divides $N\left(\mathrm{SO}_{n}\right)$. On the other hand, the natural $n$-dimensional representation $\rho$ of $\mathrm{SO}_{n}$ has $N(\rho)=2$, so $N\left(\mathrm{SO}_{n}\right)=2$.

Example 1.8. We claim that

$$
N\left(\mathrm{PSp}_{2 n}\right)= \begin{cases}2 & \text { if } n \text { is even } \\ 4 & \text { if } n \text { is odd }\end{cases}
$$

for $n \geq 2$. The number $N\left(\mathrm{PSp}_{2 n}\right)$ divides 4 and $2(n-1)$ by Example 1.2. Further, $N\left(\mathrm{PSp}_{2 n}\right)$ is even by [Merkurjev 2003, 14.2]. This shows that $N\left(\mathrm{PSp}_{2 n}\right)$ is 2 or 4 , and is 2 in case $n$ is even.

Suppose that $n$ is odd. We must show that $N(W \lambda)$ is divisible by 4 for every element $\lambda$ of the root lattice of $\operatorname{PSp}_{2 n}$. We use the same notation as [Merkurjev 2003, §14] for the weights of $\mathrm{PSp}_{2 n}$ : they are a sum $\sum_{i=1}^{n} x_{i} e_{i}$ such that $\sum x_{i}$ is even. The Weyl group $W$ is a semidirect product of $(\mathbb{Z} / 2 \mathbb{Z})^{n}$ (acting by flipping 
the signs of the $e_{i}$ ) and the symmetric group on $n$ letters (acting by permuting the $\left.e_{i}\right)$. Taking $X$ for the $(\mathbb{Z} / 2 \mathbb{Z})^{n}$-orbit of $\sum x_{i} e_{i}$, we have

$$
\frac{1}{2} \sum_{x \in X}\left\langle\sum_{i} x_{i} e_{i},\left(2 e_{n}\right)^{\vee}\right\rangle^{2}=2^{r-1} x_{n}^{2}
$$

where $r$ denotes the number of nonzero $x_{i}$ 's; see [Merkurjev 2003, proof of Lemma 14.2]. If $r=1$, then the unique nonzero $x_{i}$ is even, and we find that for $r \neq 2$, the sum - hence also $N\left(W \sum x_{i} e_{i}\right)$ - is divisible by 4 . Suppose that $x_{1}, x_{2}$ are the only nonzero $x_{i}$; then by (1.9) we have

$$
N\left(W\left(x_{1} e_{1}+x_{2} e_{2}\right)\right)= \begin{cases}2(n-1)\left(x_{1}^{2}+x_{2}^{2}\right) & \text { if } x_{1} \neq \pm x_{2} \\ 2(n-1) x_{1}^{2} & \text { if } x_{1}= \pm x_{2}\end{cases}
$$

As $n$ is odd, $N\left(W\left(x_{1} e_{1}+x_{2} e_{2}\right)\right)$ is divisible by 4 , which completes the proof of the claim.

Example 1.10. For $G$ adjoint of type $E_{7}$, we have $N(G)=12$. To see this, we note that $N(G)$ is divisible by $N(\widetilde{G})$, where $\widetilde{G}$ is the universal covering of $G$, that is, 12 divides $N(G)$. Also, $N(G)$ divides $2 h^{\vee}=36$ by Example 1.2. For the minuscule representation $\rho$ of $\widetilde{G}$, we have $\operatorname{dim} \rho=56$ and $N(\rho)=12$. The representation $\rho^{\otimes 2}$ of $\widetilde{G}$ factors through $G$ and by the derivation formula

$$
N\left(\rho_{1} \otimes \rho_{2}\right)=\left(\operatorname{dim} \rho_{1}\right) \cdot N\left(\rho_{2}\right)+\left(\operatorname{dim} \rho_{2}\right) \cdot N\left(\rho_{1}\right)
$$

(see for example [Merkurjev 2003, p. 122]) we have

$$
N\left(\rho^{\otimes 2}\right)=2(\operatorname{dim} \rho) N(\rho)=2^{6} \cdot 3 \cdot 7 .
$$

It follows that $N(G)$ equals 12 , as claimed.

\section{The Lie algebra of $G$}

2.1. Let $G$ be a split almost simple algebraic group over $F$; we fix a pinning for it. If $G_{\mathbb{Z}}$ is a split group over $\mathbb{Z}$ with the same root datum as $G$, the pinning identifies $G$ with the group obtained from $G_{\mathbb{Z}}$ by the base change $\mathbb{Z} \rightarrow F$ and the maximal torus $T$ in $G$ (from the pinning) with the base change of a maximal torus $T_{\mathbb{Z}}$ in $G_{\mathbb{Z}}$. We have a root space decomposition of the Lie algebra of $G_{\mathbb{Z}}$

$$
\operatorname{Lie}\left(G_{\mathbb{Z}}\right)=\operatorname{Lie}\left(T_{\mathbb{Z}}\right) \oplus \bigoplus_{\alpha \in R} \mathbb{Z} x_{\alpha}
$$

and

$$
\operatorname{Lie}\left(T_{\mathbb{Z}}\right)=\left\{h \in \operatorname{Lie}\left(T_{\mathbb{C}}\right) \mid \mu(h) \in \mathbb{Z} \text { for all } \mu \in T^{*}\right\},
$$


see [Steinberg 1968, p. 64]. Because $\operatorname{Lie}\left(G_{\mathbb{Z}}\right)$ is a free $\mathbb{Z}$-module, the Lie algebra $\operatorname{Lie}(G)$ of $G$ is naturally identified with $\operatorname{Lie}\left(G_{\mathbb{Z}}\right) \otimes_{\mathbb{Z}} F$, and similarly for $\operatorname{Lie}(T)$; see [Demazure and Gabriel 1970, II.4.4.8].

2.4. Write $\widetilde{G}$ for the universal covering of $G$; we use the obvious analogues of the notations in 2.1 for $\widetilde{G}$. The group $G$ acts on $\widetilde{G}$ by conjugation, hence also on $\operatorname{Lie}(\widetilde{G})$. If the kernel of the map $\widetilde{G} \rightarrow G$ is étale, then the representation $\operatorname{Lie}(\widetilde{G})$ is equivalent to the adjoint representation on $\operatorname{Lie}(G)$. In any case, the natural map $\operatorname{Lie}(\widetilde{G}) \rightarrow \operatorname{Lie}(G)$ is an isomorphism on the $F$-span of the $x_{\alpha}$.

2.5. We claim that $\operatorname{Lie}(\widetilde{G})$ is a Weyl module for $G$ in the sense of [Jantzen 2003, p. 183], that is, its character is given by Weyl's formula and it is generated as a $G$ module by a highest weight vector. The first condition holds by (2.2), so it suffices to check the second.

To check that the submodule $G x_{\tilde{\alpha}}$ generated by the highest weight vector $x_{\tilde{\alpha}}$ is all of $\operatorname{Lie}(\widetilde{G})$, one quickly reduces to checking that $G x_{\tilde{\alpha}}$ contains $\operatorname{Lie}\left(\widetilde{T}_{\mathbb{Z}}\right)$. Equation (2.3) gives a natural isomorphism $\mathbb{Z}\left[R^{\vee}\right] \stackrel{\sim}{\rightarrow} \operatorname{Lie}\left(\widetilde{T}_{\mathbb{Z}}\right)$ where $\widetilde{T}_{\mathbb{Z}}$ is the maximal torus in $\widetilde{G}_{\mathbb{Z}}$ mapping onto $T_{\mathbb{Z}}$. We write (as is usual) $h_{\alpha}$ for the image of $\alpha^{\vee}$ under this map. As $\left[x_{\alpha}, x_{-\alpha}\right]=h_{\alpha}$, the claim is proved.

2.6. See [Hiss 1984], [Hogeweij 1982, especially Corollary 2.7a], or [Steinberg 1961, §] for descriptions of the composition series of Lie $(\widetilde{G})$. They immediately give: If the characteristic of $F$ is very good for $G$, then $\operatorname{Lie}(G)$ is a simple Lie algebra. If additionally $F$ is infinite then $\operatorname{Lie}(G)$ is an irreducible $G(F)$-module.

\section{The number $E(G)$}

Definition 3.1. Maintain the notation of the preceding section. The Killing form on Lie $\left(\widetilde{G}_{\mathbb{Z}}\right)$ is divisible by $2 h^{\vee}$ [Gross and Nebe 2004] and dividing by $2 h^{\vee}$ gives an indivisible even symmetric bilinear form $\widetilde{b}$ on $\operatorname{Lie}\left(\widetilde{G}_{\mathbb{Z}}\right)$ such that

$$
\widetilde{b}\left(h_{\alpha}, h_{\alpha}\right)=2 \text { and } \widetilde{b}\left(x_{\alpha}, x_{-\alpha}\right)=1
$$

for long roots $\alpha$; see [Springer and Steinberg 1970, p. 181] or [Bourbaki 2002, Lemma VIII.2.4.3]. For a short $\operatorname{root} \beta$, we have: $\widetilde{b}\left(h_{\beta}, h_{\beta}\right)=2 c$ and $\widetilde{b}\left(x_{\beta}, x_{-\beta}\right)=c$, where $c$ is the square-length ratio of $\alpha$ to $\beta$. For example, $G=\mathrm{SL}_{n}$ has Lie algebra the trace zero $n$-by- $n$ matrices, and the form $\widetilde{b}$ is the usual trace bilinear form $(x, y) \mapsto \operatorname{trace}(x y)$; see [Bourbaki 2002, Exercise VIII.13.12].

The natural map $\operatorname{Lie}\left(\widetilde{G}_{\mathbb{Z}}\right) \rightarrow \operatorname{Lie}\left(G_{\mathbb{Z}}\right)$ is an inclusion and extending scalars to $\mathbb{Q}$ gives an isomorphism. Therefore, $\widetilde{b}$ gives a rational-valued symmetric bilinear form on $\operatorname{Lie}\left(G_{\mathbb{Z}}\right)$. We define $E(G)$ to be the smallest positive rational number such that $E(G) \cdot \widetilde{b}$ is integer-valued on $\operatorname{Lie}\left(G_{\mathbb{Z}}\right)$; we write $b$ for this form. Note that $E(G)$ is an integer by (3.2). 
Clearly, $E(G)$ depends only on the root system of $G$ and the character lattice $T^{*}$ viewed as a sublattice of the weight lattice, and not on the field $F$.

3.3. Write $\bar{G}$ for the adjoint group of $G$; we use the obvious analogues of the notations in 2.1 for $\bar{G}$. We have a commutative diagram

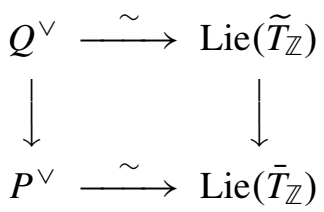

where $Q^{\vee}$ and $P^{\vee}$ are the root and weight lattices of the dual root system. The form $\widetilde{b}$ restricts to be an inner product on $Q^{\vee}$ such that the square-length of a short coroot $\alpha^{\vee}$ is 2. This inner product extends to a rational-valued inner product on $P^{\vee}$, and $E(\bar{G})$ is the smallest positive integer such that $E(\bar{G}) \cdot \widetilde{b}$ is integer-valued on $P^{\vee}$.

Example 3.4. Consider the case where $G$ is $\mathrm{PSp}_{2 n}$ for some $n \geq 2$, that is, adjoint of type $C_{n}$. In the notation of the tables in [Bourbaki 2002], the form $\widetilde{b}$ is twice the usual scalar product, $\widetilde{b}\left(e_{i}, e_{j}\right)=2 \delta_{i j}$ (Kronecker delta). The fundamental weight $\omega_{n}$ has $\widetilde{b}\left(\omega_{n}, \omega_{n}\right)=n / 2$. Checking $\widetilde{b}\left(\omega_{i}, \omega_{j}\right)$ for all $i, j$, shows that $E(\bar{G})$ is 1 if $n$ is even and 2 if $n$ is odd.

Example 3.5. Suppose that all the roots of $G$ have the same length, so that we may identify the root system $R$ with its dual and normalize lengths so that $\langle$,$\rangle is$ symmetric and equals $\widetilde{b}$ on $Q^{\vee}$.

(1) $E(\bar{G})$ is the exponent of $P / Q$, the weight lattice modulo the root lattice. Indeed, the isomorphism between $P$ and $\operatorname{Lie}\left(\bar{T}_{\mathbb{Z}}\right)$ shows that $E(\bar{G})$ is the smallest natural number such that $E(\bar{G}) \cdot\langle$,$\rangle is integer-valued on P \times P$, equivalently, the smallest natural number $e$ such that $e P$ is contained in $Q$; this is the exponent of $P / Q$.

(2) The bilinear form

$$
\widetilde{b}: \operatorname{Lie}\left(\widetilde{G}_{\mathbb{Z}}\right) \times \operatorname{Lie}\left(\bar{G}_{\mathbb{Z}}\right) \rightarrow \mathbb{Q}
$$

has image $\mathbb{Z}$ and identifies $\operatorname{Lie}\left(\bar{G}_{\mathbb{Z}}\right)$ with $\operatorname{Hom}_{\mathbb{Z}}\left(\operatorname{Lie}\left(\widetilde{G}_{\mathbb{Z}}\right), \mathbb{Z}\right)$. (On the span of the $x_{\alpha}$, this is clear from (3.2). On the Cartan subalgebras, it amounts to the statement that $\langle$,$\rangle identifies P$ with $\operatorname{Hom}(Q, \mathbb{Z})$.) It follows that $\operatorname{Lie}(\bar{G})$, as a $G$-module, is the dual of $\operatorname{Lie}(\widetilde{G})$, that is, $\operatorname{Lie}(\bar{G})$ is the module denoted by $H^{0}(\tilde{\alpha})$ in [Jantzen 2003].

Example 3.6. For $n=3$ or $n \geq 5$, we claim that $E\left(\mathrm{SO}_{n}\right)=1$.

For $n$ odd, $\mathrm{SO}_{n}$ is adjoint of type $B_{l}$ for $l=(n-1) / 2$, and we compute as in 3.3 and Example 3.4. The dual root system is of type $C_{l}$, and the form $\widetilde{b}$ is 
the usual scalar product, that is, $\widetilde{b}\left(e_{i}, e_{j}\right)=\delta_{i j}$. The fundamental weight $\omega_{i}$ is $e_{1}+e_{2}+\cdots+e_{i}$, so $E\left(\mathrm{SO}_{2 l+1}\right)=1$.

For $n$ even, $\mathrm{SO}_{n}$ has type $D_{l}$ for $l=n / 2$. The character group $T^{*}$ of a maximal torus in $\mathrm{SO}_{n}$ consists of the weights whose restriction to the center of $\mathrm{Spin}_{n}$ is 0 or agrees with the vector representation, that is, the weights $\sum c_{i} \omega_{i}$ such that $c_{l-1}+c_{l}$ is even. It follows that the cocharacter lattice $T_{*}$ is generated by the (co)root lattice and

$$
\omega_{1}=\alpha_{1}+\alpha_{2}+\cdots+\alpha_{l-2}+\frac{1}{2}\left(\alpha_{l-1}+\alpha_{l}\right)
$$

We have

$$
\widetilde{b}\left(\omega_{1}, \omega_{1}\right)=\left\langle\omega_{1}, \omega_{1}\right\rangle=1,
$$

so the form $\widetilde{b}$ is integer-valued on $T_{*}$ and $E\left(\mathrm{SO}_{2 l}\right)=1$.

Example 3.7. Let $\mathrm{HSpin}_{4 n}$ denote the image of $\operatorname{Spin}_{4 n}$ under the irreducible representation with highest weight $\omega_{l}$ for $l:=2 n$; it is a half-spin group. The character lattice $T^{*}$ consists of weights $\sum c_{i} \omega_{i}$ such that $c_{1}+c_{l-1}$ is even. The lattice generated by $Q$ and

$$
\omega_{1}+\omega_{l-1}=\frac{1}{2}\left(3 \alpha_{1}+4 \alpha_{2}+\cdots+l \alpha_{l-2}\right)+\frac{1}{4}(l+2) \alpha_{l-1}+\frac{1}{4} l \alpha_{l}
$$

contains $Q$ with index 2 and is contained in $T_{*}$, hence equals $T_{*}$. Since

$$
\widetilde{b}\left(\omega_{1}+\omega_{l-1}, \omega_{1}+\omega_{l-1}\right)=\left\langle\omega_{1}+\omega_{l-1}, \omega_{1}+\omega_{l-1}\right\rangle=\frac{3}{2}+\frac{1}{4}(l+2)=\frac{1}{2} n+2,
$$

we conclude that

$$
E\left(\mathrm{HSpin}_{4 n}\right)= \begin{cases}1 & \text { if } n \text { is even } \\ 2 & \text { if } n \text { is odd }\end{cases}
$$

\section{Formula for the trace}

The integer-valued symmetric bilinear form $b$ on $\operatorname{Lie}\left(G_{\mathbb{Z}}\right)$ defined in 3.1 gives by scalar extension a symmetric bilinear form on $\operatorname{Lie}(G)$ which we denote by $b_{F}$.

Proposition 4.1. Let $\rho$ be a representation of a split and almost simple algebraic group $G$ over $F$.

(1) $E(G)$ divides $N(\rho)$.

(2) $\operatorname{Tr}_{\rho}=\frac{N(\rho)}{E(G)} b_{F}$.

Proof. We first suppose that $F$ is the complex numbers. Write $\pi: \widetilde{G} \rightarrow G$ for the universal covering of $G$ as in Section 2. We compute $\operatorname{Tr}_{\rho \pi}$. If we decompose the representation $\rho$ with respect to the action of $\widetilde{T}$ and write $V_{\mu}$ for the eigenspace relative to the weight $\mu$, then $h_{\alpha}$ acts on $V_{\mu}$ by scalar multiplication by $\left\langle\mu, \alpha^{\vee}\right\rangle$, 
hence $\operatorname{Tr}_{\rho}\left(h_{\alpha}, h_{\alpha}\right)=\sum \operatorname{dim}\left(V_{\mu}\right)\left\langle\mu, \alpha^{\vee}\right\rangle^{2}$. By putting together the $\mu$ in an orbit $W \lambda$ (where $\lambda$ is dominant) and taking $\alpha$ to be a long root, one gets

$$
\operatorname{Tr}_{\rho \pi}\left(h_{\alpha}, h_{\alpha}\right)=2 N(\rho) .
$$

The representation $\operatorname{Lie}\left(\widetilde{G}_{\mathbb{Z}}\right) \otimes \mathbb{C}$ is irreducible and has a nondegenerate $\widetilde{G}_{\mathbb{C}}$-invariant symmetric bilinear form, so by Schur's Lemma we have

$$
\operatorname{Hom}_{\widetilde{G}_{\mathbb{C}}}\left(\operatorname{Lie}(\widetilde{G}) \otimes \mathbb{C},\left(\operatorname{Lie}(\widetilde{G})^{*}\right) \otimes \mathbb{C}\right)=\mathbb{C} .
$$

In particular, $\operatorname{Tr}_{\rho \pi}$ equals $z \widetilde{b}$ for some complex number $z$ and

$$
2 N(\rho)=\operatorname{Tr}_{\rho \pi}\left(h_{\alpha}, h_{\alpha}\right)=z \widetilde{b}\left(h_{\alpha}, h_{\alpha}\right)=2 z .
$$

Hence $\operatorname{Tr}_{\rho \pi}=N(\rho) \widetilde{b}$. (This argument can be viewed as restating pages 130131 of [Dynkin 1952].) The Lie algebra $\operatorname{Lie}\left(G_{\mathbb{Z}}\right) \otimes \mathbb{C}$ is naturally identified with $\operatorname{Lie}\left(\widetilde{G}_{\mathbb{Z}}\right) \otimes \mathbb{C}$, and (2) follows from the equation $E(G) \widetilde{b}=b$ in the case $F=\mathbb{C}$.

Now allow $F$ to be arbitrary but suppose that $\rho$ is a Weyl module. There is a $\mathbb{Z}$ form $\rho_{\mathbb{Z}}$ of $\rho$, and the form $\operatorname{Tr}_{\rho_{\mathbb{Z}}}$ is the restriction of $\operatorname{Tr}_{\rho_{\mathbb{C}}}$ on $\operatorname{Lie}\left(G_{\mathbb{C}}\right)$ to $\operatorname{Lie}\left(G_{\mathbb{Z}}\right)$. Because (2) holds over the complex numbers, it holds over the integers, and by scalar extension it holds over the field $F$ as well. Clearly the form $\operatorname{Tr}_{\rho_{\mathbb{Z}}}$ is integer valued; as $b$ is indivisible, it follows that $E(G)$ divides $N(\rho)$.

We now treat the case of an arbitrary representation $\rho$. The number $N(\rho)$ depends only on the class of $\rho$ in the representation ring $R G$. As the Weyl modules generate $R G$ as an abelian group and $E(G)$ divides $N(\psi)$ for every Weyl module $\psi$, (1) follows.

For (2), we note that the map $\rho \mapsto \operatorname{Tr}_{\rho}-(N(\rho) / E(G)) b_{F}$ is compatible with exact sequences like (1.5) in the sense that $\operatorname{Tr}_{\rho}=\operatorname{Tr}_{\rho^{\prime}}+\operatorname{Tr}_{\rho / \rho^{\prime}}$. We obtain a homomorphism of abelian groups

$$
R G \rightarrow \text { symmetric bilinear forms on } \operatorname{Lie}(G)
$$

that vanishes on the Weyl modules, hence is zero.

4.3. Because $b$ is indivisible (as a form over $\mathbb{Z}$ ), the form $b_{F}$ is not zero. Part (2) of Proposition 4.1 immediately gives:

Fact 4.4. Let $\rho$ be a representation of a split and almost simple algebraic group $G$ over $F$. Then $\operatorname{Tr}_{\rho}$ is zero if and only if the characteristic of $F$ divides $N(\rho) / E(G)$.

Furthermore, we defined $N(G)$ to be $\operatorname{gcd} N(\rho)$ as $\rho$ varies over the representations of $G$. We have proved:

Fact 4.5. Let $G$ be split and almost simple. The trace $\operatorname{Tr}_{\rho}$ is zero for every representation $\rho$ of $G$ if and only if the characteristic of $F$ divides the integer $N(G) / E(G)$. 
We have now finished half of the proof of Theorem A(2); it remains to check that the primes dividing $N(G) / E(G)$ are the primes in the rightmost column of Table I.

\section{The ratio $N(G) / E(G)$ for $G=\mathrm{SL}_{n} / \mu_{m}$}

In this section, we fix natural numbers $m$ and $n$ with $m$ dividing $n$, and we prove

Proposition 5.1. For $G=\mathrm{SL}_{n} / \boldsymbol{\mu}_{m}$, the primes dividing $N(G) / E(G)$ are precisely the primes dividing

$$
\begin{cases}\operatorname{gcd}(m, n / m) & \text { if } m \text { is odd } \\ 2 \operatorname{gcd}(m, n / m) & \text { if } m \text { is even }\end{cases}
$$

Here $\boldsymbol{\mu}_{m}$ denotes the group scheme of $m$-th roots of unity, identified with the corresponding scalar matrices in $\mathrm{SL}_{n}$.

In the important special cases where $G$ is simply connected ( $m=1), G$ is adjoint ( $m=n)$, or $n$ is square-free, the gcd in the proposition is 1 , and we have that $N(G) / E(G)$ is 1 if $m$ is odd and 2 if $m$ is even.

\section{Lemma 5.2.}

$$
E\left(\mathrm{SL}_{n} / \boldsymbol{\mu}_{m}\right)=\frac{m}{\operatorname{gcd}(m, n / m)} .
$$

Proof. Use the notation from [Bourbaki 2002] for the simple roots and fundamental weights of the root system $A_{n-1}$ of $\mathrm{SL}_{n}$. Let $\Lambda$ denote the lattice generated by the root lattice $Q$ and

$$
\beta:=\frac{n}{m} \omega_{n-1}=\frac{1}{m}\left(\alpha_{1}+2 \alpha_{2}+\cdots+(n-1) \alpha_{n-1}\right) .
$$

We claim that $\Lambda$ is identified with the cocharacter lattice $T_{*}$ for a pinning of $\mathrm{SL}_{n} / \boldsymbol{\mu}_{m}$. Certainly, $\Lambda / Q$ is cyclic of order $m$, so it suffices to check that the set of inner products $\left\langle\Lambda, T^{*}\right\rangle$ consists of integers. But $T^{*}$ is the collection of weights $\sum c_{i} \omega_{i}$ with $c_{i} \in \mathbb{Z}$ such that $\sum_{i=1}^{n-1} i c_{i}$ is divisible by $m$. We have

$$
\left\langle\beta, \sum c_{i} \omega_{i}\right\rangle=\sum_{i} \frac{1}{m} i c_{i},
$$

which is an integer when $\sum c_{i} \omega_{i}$ is in $T^{*}$, so $T_{*}=\Lambda$ as claimed.

Finally, we compute

$$
\left\langle\beta, \alpha_{n-1}\right\rangle=\frac{n}{m} \in \mathbb{Z} \quad \text { and } \quad\langle\beta, \beta\rangle=\left\langle\frac{1}{m} \sum i \alpha_{i}, \frac{n}{m} \omega_{n-1}\right\rangle=\frac{n(n-1)}{m^{2}} .
$$

Since $m$ divides $n$, it is relatively prime to $n-1$, so the minimum multiplier of $\langle$, that takes integer values on $T_{*}$ is $m / \operatorname{gcd}(m, n / m)$, as claimed. 
5.3. Weights of representations of $\mathrm{SL}_{n} / \boldsymbol{\mu}_{m}$. Fix the usual pinning of $\mathrm{SL}_{n}$, where the torus $T$ consists of diagonal matrices and the dominant weights are the maps

$$
\left(\begin{array}{ccc}
t_{1} & & \\
& \ddots & \\
& & t_{n}
\end{array}\right) \mapsto \prod_{i=1}^{n-1} t_{i}^{e_{i}}
$$

where $e_{1} \geq e_{2} \geq \cdots \geq e_{n-1} \geq 0$. Such a weight restricts to $x \mapsto x^{\sum e_{i}}$ on the center of $\mathrm{SL}_{n}$; in particular, $m$ divides $\sum e_{i}$ for every dominant weight $\lambda$ of a representation of $\mathrm{SL}_{n} / \boldsymbol{\mu}_{m}$. The proof of [Merkurjev 2003, Lemma 11.4] shows that $m$ divides $N(W \lambda)$, hence $m$ divides $N\left(\mathrm{SL}_{n} / \boldsymbol{\mu}_{m}\right)$.

5.4. We recall how to compute $N(W \lambda)$ from [Merkurjev 2003, p. 136]. Write $a_{1}>a_{2}>\cdots>a_{k-1}>a_{k}=0$ for the distinct values of the exponents $e_{i}$ in $\lambda$, where $a_{i}$ appears $r_{i}$ times, so that $n=\sum r_{i}$. We have

$$
N(W \lambda)=\frac{(n-2) !}{r_{1} ! r_{2} ! \cdots r_{k} !}\left[n\left(\sum_{i} r_{i} a_{i}^{2}\right)-\left(\sum_{i} r_{i} a_{i}\right)^{2}\right] .
$$

The dominant weight $\lambda$ with $e_{1}=m$ and $e_{i}=0$ for $i>1$ vanishes on $\boldsymbol{\mu}_{m}$ and has $N(W \lambda)=m^{2}$ by (5.5), so $N\left(\mathrm{SL}_{n} / \boldsymbol{\mu}_{m}\right)$ divides $m^{2}$.

Example 5.6. Let $\lambda$ be a dominant weight of $G$ and let $r_{i}, a_{i}$ be as in the preceding paragraph. Suppose that

$$
v_{2}\left(\sum r_{i} a_{i}\right) \geq v_{2}(n)>0,
$$

where $v_{2}(x)$ is the 2-adic valuation of $x$, that is, the exponent of the largest power of 2 dividing $x$. We claim that

$$
v_{2}(N(W \lambda))>v_{2}(n) .
$$

Write $\sum r_{i} a_{i}=2^{\theta} t$ and $n=2^{v} u$ where $\theta=v_{2}\left(\sum r_{i} a_{i}\right)$ and $v=v_{2}(n)$. Our hypothesis is that $0<v \leq \theta$. We rewrite (5.5) as

$$
N(W \lambda)=\frac{(n-2) !}{r_{1} ! r_{2} ! \cdots r_{k} !}\left[u\left(\sum_{i} r_{i} a_{i}^{2}\right)-2^{2 \theta-v} t^{2}\right] \cdot 2^{v} .
$$

Write $l$ for the minimum of $v_{2}\left(r_{i}\right)$, and fix an index $j$ such that $v_{2}\left(r_{j}\right)=l$. Note that since $\sum r_{i}=n$, we have $l \leq v \leq 2 \theta-v$.

The first term on the right side of (5.8) has 2-adic value $\geq-l$ [Merkurjev 2003, p. 137]. The term in brackets has value $\geq l$. Therefore, to prove claim (5.7), it suffices to consider the case where $v_{2}\left(\sum r_{i} a_{i}^{2}\right)=l$ and the first term on the right side of (5.8) has value $-l$; this latter condition implies that

$$
s_{2}(n-1)=s_{2}\left(r_{1}\right)+\cdots+s_{2}\left(r_{j-1}\right)+s_{2}\left(r_{j}-1\right)+s_{2}\left(r_{j+1}\right)+\cdots+s_{2}\left(r_{k}\right),
$$


where $s_{2}$ denotes the number of 1's appearing in the binary representation of the integer [Merkurjev 2003, p. 137]. That is, when adding up the numbers $r_{1}, \ldots, r_{j-1}$, $r_{j}-1, r_{j+1}, \ldots, r_{k}$ in base 2 (to get $n-1$ ), there are no carries. We check that this is impossible.

Suppose first that $l<v$. Equation (5.9) implies that there are exactly two indices, say, $j, j^{\prime}$ with $v_{2}\left(r_{j}\right)=v_{2}\left(r_{j^{\prime}}\right)=l$. As $2^{l+1}$ divides $\sum r_{i} a_{i}$, it also divides $r_{j} a_{j}+$ $r_{j^{\prime}} a_{j^{\prime}}$, hence $a_{j}$ and $a_{j^{\prime}}$ have the same parity. It follows that $2^{l+1}$ divides $r_{j} a_{j}^{2}+$ $r_{j^{\prime}} a_{j^{\prime}}^{2}$, contradicting the hypothesis that $v_{2}\left(\sum r_{i} a_{i}^{2}\right)=l$.

We are left with the case where $l=v$. By (5.9), $r_{j}$ is the unique $r_{i}$ with 2-adic valuation $l$. As $v_{2}\left(\sum r_{i} a_{i}^{2}\right)=l$, the number $a_{j}$ is odd and we have $l=v_{2}\left(\sum r_{i} a_{i}\right)=$ $\theta$. Hence both $u \cdot\left(\sum r_{i} a_{i}^{2}\right)$ and $2^{2 \theta-v} t$ have 2 -adic valuation $l$. It follows that the term in brackets in (5.8) has 2-adic valuation strictly greater than $l$, and claim (5.7) is proved.

Proof of Proposition 5.1. We write $G$ for $\mathrm{SL}_{n} / \boldsymbol{\mu}_{m}$. Paragraphs 5.3 and 5.4 give the bounds: $m$ divides $N(G)$ divides $m^{2}$. Also, $N(G)$ divides $2 n$ by Example 1.2. Applying Lemma 5.2 shows that $\operatorname{gcd}(m, n / m)$ divides $N(G) / E(G)$, which in turn divides $\operatorname{gcd}(m, n / m) \operatorname{gcd}(m, 2 n / m)$. This completes the proof for $m$ odd.

Clearly, an odd prime divides $N(G) / E(G)$ if and only if it divides $\operatorname{gcd}(m, n / m)$. So suppose that $m$ is even and 2 does not divide $\operatorname{gcd}(m, n / m)$, that is, $v_{2}(m)=$ $v_{2}(n)$. Then every dominant weight of a representation of $G$ satisfies the hypotheses of Example 5.6, hence $v_{2}(N(G))>v_{2}(n)=v_{2}(m)$. By Lemma 5.2, $v_{2}(E(G))=v_{2}(m)$, so 2 divides $N(G) / E(G)$. This completes the proof.

\section{Conclusion of proof of Theorem $\mathrm{A}(2)$}

For a split and almost simple algebraic group $G$, we now verify that the primes dividing $N(G) / E(G)$ are those in the last column of Table I. Together with 4.5, this will prove Theorem $\mathrm{A}(2)$.

For $G$ simply connected, $E(G)$ is 1 and $N(G)$ is divisible precisely by the torsion primes of $G$, see 1.6. We assume that $G$ is not simply connected and write $\widetilde{G}$ for the universal covering of $G$; obviously $N(\widetilde{G})$ divides $N(G)$.

For $G=\mathrm{PSp}_{2 n}, \mathrm{SO}_{n}$, or adjoint of type $E_{7}$, one combines Examples 1.8 and 3.4, 1.7 and 3.6, or 1.10 and 3.5, respectively.

For $G$ adjoint of type $D_{n}$, we have $E(G)=2$ by Example 3.5. Also, 4 divides $N(G)$ by [Merkurjev 2003, 15.2]. On the other hand, the spinor representations of $\widetilde{G}$ have Dynkin index $2^{n-3}$ [Laszlo and Sorger 1997], and it is easy to use this as in Example 1.10 to construct a representation $\rho$ of $G$ with $N(\rho)$ a power of 2 . This shows that $N(G) / E(G)$ is a power of 2 and is not 1 .

Now let $G=\mathrm{HSpin}_{4 n}$ for some $n \geq 3$. The dual of the center of $\operatorname{Spin}_{4 n}$ is the Klein four-group, and we write $\chi$ for the unique nonzero element that vanishes 
on the kernel of the map $\operatorname{Spin}_{4 n} \rightarrow \mathrm{HSpin}_{4 n}$. The gcd of $N(W \lambda)$ as $\lambda$ varies over the weights that restrict to $\chi$ (respectively, 0) on the center of $\operatorname{Spin}_{4 n}$ is $2^{2 n-3}$ (respectively, divisible by 4) by [Merkurjev 2003, p. 146], hence $N(G)$ is a power of 2 and at least 4 . On the other hand, $E\left(\mathrm{HSpin}_{4 n}\right)$ is 1 or 2 . We conclude that $N(G) / E(G)$ is a power of 2 and is not 1 .

For $G$ adjoint of type $E_{6}$, the number $N(G)$ is divisible by $N(\widetilde{G})=6$ and divides $2 h^{\vee}=24$ by Example 1.2. By Example 3.5, $N(G) / E(G)$ is 2, 4, or 8 . This completes the proof of Theorem A(2).

Example 6.1. Suppose that the characteristic of $F$ is an odd prime $p$, and let $n$ be a natural number divisible by $p^{2}$. Every trace form of $\mathrm{SL}_{n} / \boldsymbol{\mu}_{p}$ is zero by Theorem $\mathrm{A}(2)$, even though the universal covering $\mathrm{SL}_{n}$ and adjoint group $\mathrm{PGL}_{n}$ have representations with nonzero trace forms.

\section{Proof of Theorem A(1)}

We now prove Theorem $\mathrm{A}(1)$; we show that the following three statements are equivalent:

(a) The characteristic of $F$ is very good for $G$.

(b) $\operatorname{Lie}(G)$ is a simple algebra and there is a representation $\rho$ of $G$ with $\operatorname{Tr}_{\rho}$ nonzero.

(c) There is a representation $\rho$ of $G$ with $\operatorname{Tr}_{\rho}$ nondegenerate.

Suppose (a) holds. Then $\operatorname{Lie}(G)$ is simple, as in 2.6. The existence of a representation $\rho$ with nonzero trace follows from Theorem $\mathrm{A}(2)$, so (b) holds. It is easy to check that for a representation $\psi$ of $\operatorname{Lie}(G), \operatorname{Tr}_{\psi}([x, y], z)=\operatorname{Tr}_{\psi}(x,[y, z])$ for all $x, y, z \in \operatorname{Lie}(G)$. So the radical of a trace form on $\operatorname{Lie}(G)$ is an ideal, and (b) implies (c).

Now suppose that (a) fails; we check that (c) also fails. By Theorem A(2), we only need to consider those cases where the characteristic of $F$ appears in the middle column of Table I and not in the right column, namely the cases:

(i) $G$ has type $G_{2}$ and $\operatorname{char} F=3$, or $G$ is $\mathrm{Sp}_{2 n}$ and $\operatorname{char} F=2$, or $G$ is $\mathrm{SL}_{n} / \boldsymbol{\mu}_{m}$ and $\operatorname{char} F$ is odd and divides $n / m$ but not $m$.

(ii) $G$ is adjoint of type $E_{6}$ and $\operatorname{char} F=3$, or $G$ is $\mathrm{SL}_{n} / \boldsymbol{\mu}_{m}$ and $\operatorname{char} F \operatorname{divides} m$ but not $n / m$.

We write $\pi: \widetilde{G} \rightarrow G$ for the universal covering of $G$. In case (i), the kernel of $\pi$ is étale, so $\operatorname{Lie}(G)$ is a Weyl module by 2.5. For all three of the types listed, $\operatorname{Lie}(G)$ has a nontrivial submodule $M$, namely the subalgebra generated by the short roots (for $G_{2}$ ) or the center (in the other two cases). It follows that $M$ is contained in the radical of $\operatorname{Tr}_{\rho}$ - see [Garibaldi 2008, 6.2], for example - hence (c) fails. 
In case (ii), every representation $\rho$ of $G$ gives a representation $\rho \pi$ of $\widetilde{G}$ whose trace form $\operatorname{Tr}_{\rho \pi}$ vanishes on $\operatorname{Lie}(\widetilde{G})$ by Theorem A(2) (for $E_{6}$ ) or 5.3 (for $\mathrm{SL}_{n}$ ). Hence the image of $\mathrm{d} \pi$ is a totally isotropic subspace for $\operatorname{Tr}_{\rho}$. As

$$
\operatorname{dim}(\operatorname{imd} \pi)=\operatorname{dim} \widetilde{G}-\operatorname{dim}(\operatorname{ker} \mathrm{d} \pi)=\operatorname{dim} G-1
$$

is strictly greater than half the dimension of $G$, the form $\operatorname{Tr}_{\rho}$ is degenerate and (c) fails. This concludes the proof of Theorem A(1).

\section{Richardson's condition}

In the literature, the weak version of the "if" direction of Theorem A(1) is used to deduce Richardson's condition from [Richardson 1967, p. 3]. Our slightly finer version of the "if" direction gives a slightly finer version of Richardson's condition; we state it here for the convenience of the reader. As in Theorem A, $G$ is a split almost simple algebraic group over a field $F$.

Proposition 8.1. If the characteristic of $F$ is very good for $G$, then there is a representation $\rho: G \rightarrow \mathrm{GL}(V)$ such that $\mathrm{d} \rho$ is an injection $\operatorname{Lie}(G) \hookrightarrow \mathfrak{g l}(V)$ and there is a subspace $M$ of $V$ such that $\mathfrak{g l}(V)=\mathrm{d} \rho(\operatorname{Lie}(G)) \oplus M, \operatorname{Id}_{V}$ is in $M$, and $\operatorname{Ad}(\rho(G)) M \subseteq M$.

Proof. Theorem A(1) gives a representation $\rho$ so that $\operatorname{Tr}_{\rho}$ is nondegenerate. In particular, the restriction of the symmetic bilinear form $(x, y) \mapsto \operatorname{trace}(x y)$ on $\mathfrak{g l}(V)$ to $\mathrm{d} \rho(\operatorname{Lie}(G))$ is nondegenerate. (And obviously $\mathrm{d} \rho$ must be injective.)

Take $M$ to be the space of $x \in \mathfrak{g l}(V)$ such that $\operatorname{trace}(\mathrm{d} \rho(\operatorname{Lie}(G)) x)=0$. Trivially, $M$ is invariant under $\operatorname{Ad}(\rho(G))$. Nondegeneracy of $\operatorname{Tr}_{\rho}$ shows that $M$ meets $\mathrm{d} \rho(\operatorname{Lie}(G))$ only at 0 , and dimension count shows that $V=\mathrm{d} \rho(\operatorname{Lie}(G)) \oplus M$. As $G$ is semisimple, the image $\rho(G)$ is contained in $\operatorname{SL}(V)$, hence $\mathrm{d} \rho(\operatorname{Lie}(G))$ lies in $\mathfrak{s l}(V)$, that is, $\operatorname{Id}_{V}$ belongs to $M$.

The proposition is essentially known, but the usual argument as in [Richardson 1967, §5], [Jantzen 2004, 2.6], [Humphreys 1995, p. 48], or [Springer and Steinberg 1970, p. 184] is different. For example, the usual approach to treating an adjoint group $G$ of type $C_{n}$ or $D_{n}$ replaces $G$ with its covering $G^{\prime}=\mathrm{Sp}_{2 n}$ or $\mathrm{SO}_{2 n}$ and then gives a representation of $G^{\prime}$ with the desired properties.

\section{Complements: Characteristic 2}

In characteristic 2 , one might prefer to consider, instead of the symmetric bilinear form $\operatorname{Tr}_{\rho}$, the quadratic form

$$
s_{\rho}: x \mapsto-\operatorname{trace}\left(\bigwedge^{2} \mathrm{~d} \rho(x)\right),
$$


which gives the negative of the degree 2 coefficient of the characteristic polynomial of $\mathrm{d} \rho(x)$. (Because $\mathrm{d} \rho(\operatorname{Lie}(G))$ consists of trace zero matrices, $s_{\rho}$ is the map $x \mapsto$ $\operatorname{trace}\left(\mathrm{d} \rho(x)^{2}\right) / 2$; our definition has the advantage that it obviously makes sense in characteristic 2 as well.) The bilinear form derived from $s_{\rho}$, that is, $(x, y) \mapsto$ $s_{\rho}(x+y)-s_{\rho}(x)-s_{\rho}(y)$, is $\operatorname{Tr}_{\rho}$.

Theorem $\mathrm{A}(2)$ is easy to extend. In case $G$ is simply connected, $\operatorname{Lie}(G)$ is a Weyl module by 2.5 and $s_{\rho}$ is zero if and only if $\operatorname{Tr}_{\rho}$ is zero by [Garibaldi 2008, Proposition 6.4(1)]. That is, the conditions in Theorem A(2) are equivalent to: For every representation $\rho$ of $G$, the quadratic form $s_{\rho}$ is zero. (This is true in all characteristics but is only nontrivial in characteristic 2 .)

Alternatively, one can proceed as follows. The bilinear form $\widetilde{b}$ on $\operatorname{Lie}\left(\widetilde{G}_{\mathbb{Z}}\right)$ is even [Gross and Nebe 2004, Proposition 4], so it is the bilinear form derived from a unique quadratic form $\widetilde{q}$ on $\operatorname{Lie}\left(\widetilde{G}_{\mathbb{Z}}\right)$. The form $\widetilde{q}$ extends to a rational-valued quadratic form on $\operatorname{Lie}\left(G_{\mathbb{Z}}\right)$ and we write $E_{q}(G)$ for the smallest positive rational number such that $E_{q}(G) \widetilde{q}$ is integer-valued on $\operatorname{Lie}\left(G_{\mathbb{Z}}\right)$. The number $E_{q}(G)$ is $E(G)$ or $2 E(G)$, and both cases can occur. (For example, take $G=\widetilde{G}$ or $\mathrm{SO}_{2 l}$, respectively.) The statements and proofs of 4.4 and 4.5 go through if we replace $\operatorname{Tr}_{\rho}, E(G)$, and $b$ with $s_{\rho}, E_{q}(G)$, and $E_{q}(G) \widetilde{q}$ respectively.

\section{Complements: Nonsplit groups}

We can extend our results above to the case where $G$ is not split, that is, we can replace the hypotheses " $G$ is split and almost simple" with " $G$ is absolutely almost simple". Indeed, suppose that $G$ is absolutely almost simple over $F$, that is, there is a split and almost simple group $G^{\prime}$ over $F$ and an isomorphism $f: G^{\prime} \rightarrow G$ defined over a separable closure $F_{\text {sep }}$ of $F$. Fix a pinning for $G^{\prime}$ and write $b^{\prime}$ for the indivisible bilinear form on $\operatorname{Lie}\left(G_{\mathbb{Z}}^{\prime}\right)$ defined in 3.1. Clearly, the automorphism group of $G^{\prime}$, which is an affine group scheme over $\mathbb{Z}$, leaves $b^{\prime}$ invariant, so it maps into the orthogonal group of $b^{\prime}$. Galois descent (via $f$ ) gives a $G$-invariant symmetric bilinear form $b_{F}$ on $\operatorname{Lie}(G)$ such that the differential $\mathrm{d} f$ identifies $b_{F}^{\prime} \otimes$ $F_{\text {sep }}$ with $b_{F} \otimes F_{\text {sep }}$.

Given a representation $\rho$ of $G$ over $F$, we get a representation $\rho f$ of $G^{\prime}$ over $F_{\text {sep }}$ and an integer $N(\rho f)$ defined in 1.3; put $N(\rho):=N(\rho f)$. (In the special case where $G$ is split over $F$, this agrees with our previous definition.) We define $N(G)$ as in (1.4); it is the gcd of $N(\rho)$ as $\rho$ varies over the representations of $G$ defined over $F$. Obviously, $N(G)$ is divisible by $N\left(G_{F_{\text {sep }}}\right)$, that is, $N\left(G^{\prime}\right)$, and it depends on the field $F$.

We put $E(G):=E\left(G^{\prime}\right)$. It does not depend on the field $F$.

With these definitions for $N(G)$ and $E(G)$, conclusions (1) and (2) of Proposition 4.1 hold for absolutely almost simple $G$. Indeed, it suffices to check them 
over $F_{\text {sep }}$, where they hold by the original version of the proposition. It follows immediately that the conclusions of 4.4 and 4.5 hold for every absolutely almost simple group $G$.

We now extend Theorem A. Recall that there is a natural action of the absolute Galois group $\operatorname{Gal}(F)$ of $F$ on the Dynkin diagram $\Delta$ of $G$ [Tits 1966, 2.3]. As in [Tits 1966, p. 54], we say, for example, that $G$ has type ${ }^{3} D_{4}$ if $\Delta$ has type $D_{4}$ and the image of the map $\operatorname{Gal}(F) \rightarrow \operatorname{Aut}(\Delta)$ has order 3. We say that the characteristic of $F$ is not very good for $G$ if and only if it is not very good for the corresponding split group $G^{\prime}$; these primes are listed in the middle column of Table I.

Theorem $\mathbf{A}^{\prime}$. Let $G$ be an absolutely almost simple algebraic group over a field $F$.

(1) Every representation $\rho$ of $G$ over $F$ has $\operatorname{Tr}_{\rho}$ degenerate if and only if the characteristic of $F$

$$
\begin{cases}\text { divides } 2 n & \text { if } G \text { has type }{ }^{2} A_{n-1} \text { for some odd } n \geq 3 \\ \text { is } 2 \text { or } 3 & \text { if } G \text { has type }{ }^{3} D_{4} \text { or }{ }^{6} D_{4} \\ \text { is not very good for } G & \text { otherwise. }\end{cases}
$$

(2) Suppose G is not simply connected and not of type A. Every representation of $G$ has $\operatorname{Tr}_{\rho}$ zero if and only if the characteristic of $F$ is as in the table:

\begin{tabular}{|c|c|}
\hline type of $G$ & $\operatorname{char} F$ \\
\hline$B_{n}(n \geq 3) ; C_{n}(n \geq 2) ;{ }^{1} D_{n}$ or ${ }^{2} D_{n}(n \geq 4) ;$ or $E_{6}$ & 2 \\
${ }^{3} D_{4},{ }^{6} D_{4}, E_{7}$ & 2 or 3 \\
\hline
\end{tabular}

Regarding the omitted cases in part (2), for $G$ simply connected, the number $E(G)$ is 1 , so every representation $\rho$ of $G$ has $\operatorname{Tr}_{\rho}$ zero if and only if the characteristic divides $N(G)$ by 4.5 ; this number (using that $G$ is simply connected) is calculated in [Merkurjev 2003, $\S 11-16]$. We leave the type $A$ case of (2) as an exercise for the reader.

Proof of Theorem $A^{\prime}$. To prove (2), by 4.5 it remains to show that the primes in the table are those dividing $N(G) / E(G)$. As $N\left(G^{\prime}\right)$ divides $N(G)$ and $E\left(G^{\prime}\right)$ equals $E(G)$, we have the trivial equation

$$
\frac{N(G)}{E(G)}=\frac{N(G)}{N\left(G^{\prime}\right)} \frac{N\left(G^{\prime}\right)}{E\left(G^{\prime}\right)}
$$

where all three terms are integers. The primes dividing $N\left(G^{\prime}\right) / E\left(G^{\prime}\right)$ are listed in Table I, so it suffices to check which primes divide $N(G) / N\left(G^{\prime}\right)$ and are not in that table. 
For $G$ adjoint of type $E_{6}$, the proof that $N(G) / E(G)$ is a power of 2 from the end of Section 6 goes through without change.

The proof of [Merkurjev 2003,10.11] shows that every prime dividing $N(G) / N(G)$ divides the exponent of $P / Q$ (the weight lattice modulo the root lattice) or the order of the image of $\operatorname{Gal}(F) \rightarrow \operatorname{Aut}(\Delta)$. For $G$ of type $B_{n}(n \geq 3), C_{n}(n \geq 2),{ }^{1} D_{n}$ or ${ }^{2} D_{n}(n \geq 4)$, or $E_{7}$, the exponent of $P / Q$ is 2 and the image of $\operatorname{Gal}(F) \rightarrow \operatorname{Aut}(\Delta)$ has order at most 2. As 2 divides $N\left(G^{\prime}\right) / E\left(G^{\prime}\right)$, part (2) is proved for these groups.

For $G$ adjoint of type ${ }^{3} D_{4}$ or ${ }^{6} D_{4}$, write $\widetilde{G} \rightarrow G$ for the universal covering of $G$. The number $N(\widetilde{G})$ is 6 or 12 by [Merkurjev 2003, 16.5] and divides $N(G)$. As $E(G)$ is 2 by Example 3.5, $N(G) / E(G)$ is divisible by 3. Part (2) of the theorem is proved.

(We remark that applying the argument from the two previous paragraphs in the case where $G$ has type $A_{n-1}$ shows that every prime dividing $N(G) / N\left(G^{\prime}\right)$ divides $2 n$. If $n$ is odd and $\geq 3$ and $G$ has type ${ }^{2} A_{n-1}$, then 2 divides $N(\widetilde{G})$ by [Merkurjev $2003,12.6]$ hence also $N(G)$, yet $E(G)$ is odd by Lemma 5.2 , so $N(G) / E(G)$ is even.)

We now prove part (1) by imitating Section 7. We replace (a) with the condition that the characteristic of $F$ is not as in the statement of Theorem $\mathrm{A}^{\prime}(1)$; we denote this condition by (a)'.

Suppose that (a)' holds. The characteristic is very good for $G$ and $\operatorname{Lie}(G) \otimes F_{\text {sep }}$ is simple as in 2.6, hence $\operatorname{Lie}(G)$ is simple. If $G$ is neither simply connected nor of type $A$, then there is a representation $\rho$ of $G$ with $\operatorname{Tr}_{\rho}$ nonzero by part (2) and (b) holds. If $G$ is simply connected, then checking [Merkurjev 2003] verifies that $N(G)$ is not divisible by the characteristic and again (b) holds. In the remaining case where $G$ has type $A$, the characteristic does not divide $N\left(G^{\prime}\right)$ by 5.4 nor does it divide $N(G) / N\left(G^{\prime}\right)$ by the discussion above; by (10.1), we find that (b) holds.

As in Section 7, (b) trivially implies (c).

Finally, suppose that (a)' fails; we will show that (c) fails. We assume that the characteristic is very good, otherwise (c) fails because it does so over $F_{\text {sep }}$. That is, we are in one of the cases

(i) $\operatorname{char} F=3$ and $G$ has type ${ }^{3} D_{4}$ or ${ }^{6} D_{4}$; or

(ii) $\operatorname{char} F=2$ and $G$ has type ${ }^{2} A_{n-1}$ for some odd $n$.

But in these cases the characteristic divides $N(G) / E(G)$ by the proof of part (2) above, and (c) fails.

Example 10.2. Let $F$ be a field of prime characteristic $p$ with a central division $F$-algebra $A$ of degree $p$. Take $G$ to be the group $\operatorname{SL}(A)$ whose $F$-points are the elements of $A$ with determinant 1. This group is simply connected, so $N(G) / E(G)$ is $p$ by [Merkurjev 2003, 11.5]. That is, $\operatorname{Tr}_{\rho}$ is zero for every representation $\rho$ of $G$ over $F$. On the other hand, $N\left(G_{F_{\text {sep }}}\right)$ is 1 , so there are representations of $G$ defined 
over $F_{\text {sep }}$ (for example, the natural representation of $\mathrm{SL}_{n}$ ) that have a trace form that is not zero.

A similar statement holds for groups of type ${ }^{3} D_{4}$ or ${ }^{6} D_{4}$ over fields of characteristic 3.

\section{Trace forms and Lie algebras}

This section collects some results regarding $\operatorname{Tr}_{\psi}$, where $\psi$ is a representation of the Lie algebra of an algebraic group $G$ and we do not assume that $\psi$ is the differential of a representation of $G$.

11.1. Fix a positive integer $n$ and assume that the characteristic of $F$ is a prime dividing $n$ and $\neq 2,3$. The Lie algebra $\mathfrak{s l}_{n}$ of trace zero $n$-by- $n$ matrices has center $\mathfrak{c}$ the scalar matrices and $\mathfrak{s l}_{n} / \mathfrak{c}$ is simple [Steinberg 1961, 2.6]. We give a new proof of:

Proposition 11.2 [Block 1962, Theorem 6.2]. Under the hypotheses of 11.1, every representation of $\mathfrak{s l}_{n} / \mathfrak{c}$ has zero trace form.

Proof. For sake of contradiction, suppose that there is an irreducible representation $\psi$ of $\mathfrak{s l}_{n} / \mathfrak{c}$ with nonzero trace form. Then $\psi$ is restricted by [Block 1962, Theorem 5.1], using that $F$ has characteristic $\neq 2,3$. The composition of $\psi$ with $\mathfrak{s l}_{n} \rightarrow \mathfrak{s l}_{n} / \mathfrak{c}$ is a restricted irreducible representation of $\mathfrak{s l}_{n}$, which is the differential of a representation $\rho$ of $\mathrm{SL}_{n}$ by [Curtis 1960; Steinberg 1963].

By construction $\operatorname{Tr}_{\rho}$ is not zero and $\mathrm{d} \rho$ vanishes on the scalar matrices. Identifying the center of $\mathrm{SL}_{n}$ with the (nonreduced) group scheme $\boldsymbol{\mu}_{n}$ identifies the restriction of $\rho$ to $\boldsymbol{\mu}_{n}$ with a map $x \mapsto x^{l}$. Our hypothesis on $\mathrm{d} \rho$ says that $l$ is divisible by the characteristic $p$ of $F$, hence $\rho$ factors through the natural map $\mathrm{SL}_{n} \rightarrow \mathrm{SL}_{n} / \boldsymbol{\mu}_{p}$. Paragraph 5.3 says that $N(\rho)$ is divisible by $p$, hence $\operatorname{Tr}_{\rho}$ vanishes by 4.4 , a contradiction.

Since every irreducible representation has zero trace form, the same holds for every representation like at the end of the proof of Proposition 4.1.

11.3. Proof of Theorem $B$. Let $G$ be a group of type $E_{8}$, and suppose that there is a representation $\psi$ of $\operatorname{Lie}(G)$ such that $\operatorname{Tr}_{\psi}$ is not zero. We may assume that $\psi$ is irreducible. Then the Block-Premet theorem on page 563 implies that $\psi$ is restricted, hence is the differential of a representation of $G$. Theorem $\mathrm{A}(2)$ implies that the characteristic of $F$ is $\neq 2,3,5$.

We close by proving that over a field of characteristic 5 , the Lie algebra of a group of type $E_{8}$ has no quotient trace form. For a Lie algebra $L$ over $F$ and a representation $\psi$ of $L$, write $\operatorname{rad} \psi$ for the radical of the trace bilinear form $\operatorname{Tr}_{\psi}$; it is an ideal of $L$. 
Corollary 11.4. For every representation $\psi$ of every Lie algebra $L$ over a field of characteristic 5, the quotient $L / \operatorname{rad} \psi$ is not isomorphic to the Lie algebra of an algebraic group of type $E_{8}$.

Proof. Suppose the corollary is false. That is, suppose that there is a group $G$ of type $E_{8}$ and a Lie algebra $L$ with a representation $\psi$ and a surjection $\pi: L \rightarrow \operatorname{Lie}(G)$ with kernel the radical of $\operatorname{Tr}_{\psi}$.

By [Block 1962, Lemma 2.1] — using that the characteristic is $\neq 2,3$ - we may assume that the radical of $\operatorname{Tr}_{\psi}$ is contained in the center of $L$, that is, $L$ is a central extension of $\operatorname{Lie}(G)$. It follows that there is a map $f: \operatorname{Lie}(G) \rightarrow L$ such that $\pi f$ is the identity [Steinberg 1962, Theorem 6.1(c)]. Clearly, the representation $\psi f$ of $\operatorname{Lie}(G)$ has nonzero trace form. As in the proof of Proposition 11.2, we deduce that $G$ has a representation $\rho$ such that $\operatorname{Tr}_{\rho}$ is not zero, but this is impossible by Theorem A(2).

\section{Acknowledgments}

It is a pleasure to thank Jean-Pierre Serre for numerous helpful discussions and for pointing out the utility of the form $\widetilde{b}$ (from [Gross and Nebe 2004], see 3.1) early in this project. I also thank George McNinch and Burt Totaro for their comments, the NSF under grant DMS-0653502 for its support, and the Institut des Hautes Etudes Scientifiques for its hospitality.

\section{Appendix: On trace forms of Lie algebras of type $E_{8}$}

by Alexander Premet

All basic notions and results of modular Lie theory used in this appendix can be found in [Premet 1995] and references therein.

Let $G$ be an algebraic group of type $E_{8}$ over an algebraically closed field of characteristic $p>0$ and $\mathfrak{g}=\operatorname{Lie}(G)$. It is well known that $\mathfrak{g}$ is a simple Lie algebra carrying an $(\operatorname{Ad} G)$-equivariant $[p]$-th power map $x \mapsto x^{[p]}$. Since the universal enveloping algebra $U(\mathfrak{g})$ is a finite module over its central subalgebra generated by all $x^{p}-x^{[p]}$ with $x \in \mathfrak{g}$, all irreducible $\mathfrak{g}$-modules are finite dimensional. Furthermore, for every irreducible $\mathfrak{g}$-module $M$ there is a linear function $\xi=\xi_{M}$ on $\mathfrak{g}$ such that $x^{p}-x^{[p]}$ acts on $M$ as the scalar operator $\xi(x)^{p} \operatorname{Id}_{M}$. The function $\xi_{M}$ is called the $p$-character of $M$. Denote by $I_{\xi}$ the two-sided ideal of $U(\mathfrak{g})$ generated by all elements $x^{p}-x^{[p]}-\xi(x)^{p}$, where $x \in \mathfrak{g}$. The factor-algebra of $U(\mathfrak{g}) / I_{\xi}$ is called the reduced enveloping algebra associated with $\xi$ and denoted $U_{\xi}(\mathfrak{g})$. It has dimension $p^{\operatorname{dim} \mathfrak{g}}$. Clearly, $M$ is a $U_{\xi}(\mathfrak{g})$-module. We say that $M$ is restricted if $\xi_{M}=0$. 
For $p>3$, the theorem below was first proved in [Block 1962]. The aim of this appendix is to give a proof valid in any positive characteristic.

Theorem. If $\psi: \mathfrak{g} \rightarrow \mathfrak{g l}(V)$ is an irreducible representation with $\operatorname{Tr}_{\psi} \neq 0$, then $V$ is a restricted $\mathfrak{g}$-module.

Proof. Suppose $\psi$ is not restricted and let $\chi$ be the $p$-character of $V$. Then $\chi$ is a nonzero linear function on $\mathfrak{g}$. We show that $\operatorname{Tr}_{\psi}$ is zero.

Let $T$ be a maximal torus of $G$ and $\mathfrak{t}:=\operatorname{Lie}(T)$. As in sections 1 and 2, we write $R$ for the root system of $G$ relative to $T$ and $h_{\alpha}$ for the image of the coroot $\alpha^{\vee}$ in $\mathfrak{t}:=\operatorname{Lie}(T)$. (In our case, the group $G$ is both adjoint and simply connected.) Since $\mathfrak{g}$ is an irreducible ( $\operatorname{Ad} G$ )-module, every nonzero adjoint $G$-orbit spans $\mathfrak{g}$. Thus, replacing $\mathfrak{t}$ by its $G$-conjugate if necessary, we may assume that $\chi\left(h_{\beta}\right) \neq 0$ for some $\beta \in R$.

There are root vectors $e_{ \pm \beta} \in \mathfrak{g}^{ \pm \beta}$ such that $\mathfrak{s}:=F e_{-\beta} \oplus F h_{\beta} \oplus F e_{\beta}$ is isomorphic to $\mathfrak{s l}_{2}$. Replacing $\mathfrak{t}$ by its conjugate $\left(\operatorname{Ad} x_{-\beta}(\lambda)\right)(\mathfrak{t})$ for a suitable $x_{-\beta}(\lambda)$ in the unipotent root subgroup $U_{-\beta}$ of $G$, we may assume without loss of generality that $\left.\chi\right|_{\mathfrak{s}} \neq 0$ and $\chi\left(e_{\beta}\right)=0$. Then every $\mathfrak{s}$-composition factor $M$ of $V$ is a baby Verma module, that is, $M \cong Z_{\xi}(a)$, where $\xi=\left.\chi\right|_{\mathfrak{s}}$ and $a \in F$ is a root of the equation $X^{p}-X=\xi\left(h_{\alpha}\right)^{p}$. Note that $\operatorname{dim} M=p$, the operator $h_{\beta}$ acts semisimply on $M$, and the $h_{\beta}$-weights of $M$ are $a, a-2, \ldots, a-2(p-1)$.

First suppose $p>3$. Then $\operatorname{trace}_{M}\left(h_{\beta}^{2}\right)=\sum_{i=0}^{p-1}(a-2 i)^{2}=p a^{2}-2 a p(p-1)+$ $\frac{2}{3}(p-1) p(2 p-1)=0$. Since this holds for every $\mathfrak{s}$-composition factor $M$ of $V$, we obtain $\operatorname{Tr}_{\psi}\left(h_{\beta}, h_{\beta}\right)=0$. As $\mathfrak{g}$ is a simple Lie algebra and $\operatorname{Tr}_{\psi}$ is $\mathfrak{g}$-invariant, $\operatorname{Tr}_{\psi}$ is a multiple of the form $b_{F}$ from Section 4. Hence $\operatorname{Tr}_{\psi}$ is zero.

Next suppose $p=3$. Then the $h_{\beta}$-weights of $M$ are $a, a+1, a-1$, hence $\operatorname{trace}_{M}\left(h_{\beta}^{2}\right)=a^{2}+(a+1)^{2}+(a-1)^{2}=2$. It follows that $\operatorname{trace}_{M}\left(h_{\beta}^{2}\right)$ is independent of $M$. Since all $\mathfrak{s}$-composition factors of $V$ are three-dimensional, we deduce that $\operatorname{Tr}_{\psi}\left(h_{\beta}, h_{\beta}\right)=2(\operatorname{dim} V) / 3$. Note that $\mathfrak{s}$ can be included into a Levi subalgebra of type $A_{7}$; call it $\mathfrak{l}$. Since $\mathfrak{s} \subset \mathfrak{l}$, all $\mathfrak{l}$-composition factors of $V$ have the same nonzero p-character. But then the Kac-Weisfeiler conjecture, which thanks to [Premet 1995, Theorem 3.10] holds for $\mathfrak{s l}_{8}$ in characteristic 3, implies that all such factors have dimension divisible by 9 . Then 9 divides $\operatorname{dim} V$, forcing $\operatorname{Tr}_{\psi}\left(h_{\beta}, h_{\beta}\right)=0$. As in the $p>3$ case, $\operatorname{Tr}_{\psi}$ is zero.

Finally, suppose $p=2$. Then the $\mathfrak{s l}_{2}$-algebra $\mathfrak{s}=F e \oplus F h \oplus F f$ is nilpotent and $h$ lies in the center of $\mathfrak{s}$. However, the reduced enveloping algebra $U_{\xi}(\mathfrak{s})$ is semisimple whenever $\xi(h) \neq 0$. Indeed, $U_{\xi}(\mathfrak{s})$ then possesses two nonequivalent two-dimensional irreducible modules, $M$ and $N$, induced from one-dimensional modules over a Borel subalgebra of $\mathfrak{s}$. The central element $h$ of $\mathfrak{s}$ acts on $M$ and $N$ by different scalars. There are exactly two choices here, namely, $a$ and $a+1$, where $a$ is a root of the equation $X^{2}-X=\xi(h)^{2}$. As a consequence, $U_{\xi}(\mathfrak{s})$ maps onto 
a direct sum of two copies of $\operatorname{Mat}_{2}(F)$. Since $\operatorname{dim} U_{\xi}(\mathfrak{s})=2^{3}=8$, this map is an isomorphism. Thus, $U_{\xi}(\mathfrak{s})$ is semisimple with two isoclasses of simple modules, both of which are two-dimensional.

Suppose now that we have found two commuting $\mathfrak{s l}_{2}$-subalgebras $\mathfrak{s}_{i}=F e_{i} \oplus$ $F h_{i} \oplus F f_{i}$ in $\mathfrak{g}$, where $i=1,2$, such that

(a) the sum $\mathfrak{s}_{1}+\mathfrak{s}_{2}$ is direct;

(b) $\chi\left(h_{i}\right) \neq 0$ for $i=1,2$;

(c) $e_{1} \in \mathfrak{g}^{\gamma}$ and $f_{1} \in \mathfrak{g}^{-\gamma}$ for some $\gamma \in R$.

Our preceding remark then would show that $V$ is a semisimple module over the subalgebra $U_{\chi}\left(\mathfrak{s}_{1} \oplus \mathfrak{s}_{2}\right) \cong U_{\chi}\left(\mathfrak{s}_{1}\right) \otimes U_{\chi}\left(\mathfrak{s}_{2}\right)$ of $U_{\chi}(\mathfrak{g})$ (to ease notation, we identify $\chi$ with its restriction to $\left.\mathfrak{s}_{i}, i=1,2\right)$. Let $M$ and $N$ be two irreducibles for $U_{\chi}\left(\mathfrak{s}_{1}\right)$ described earlier. Then $V$ decomposes as a tensor product $V=(M \otimes P) \bigoplus(N \otimes Q)$ for some semisimple $U_{\chi}\left(\mathfrak{s}_{2}\right)$-modules $P$ and $Q$. Therefore,

$$
\operatorname{Tr}_{\psi}\left(e_{1}, f_{1}\right)=r \operatorname{dim} P+s \operatorname{dim} Q,
$$

where $r=\operatorname{trace}_{M}\left(e_{1} f_{1}\right)$ and $s=\operatorname{trace}_{N}\left(e_{1} f_{1}\right)$. As both $P$ and $Q$ must have even dimension by our preceding remark, this would yield $\operatorname{Tr}_{\psi}\left(\mathfrak{g}^{\gamma}, \mathfrak{g}^{-\gamma}\right)=0$. Hence $\operatorname{Tr}_{\psi}=0$ by (3.2), using that $\gamma$ is (trivially) a long root.

So it remains to find two commuting $\mathfrak{s l}_{2}$-triples as above. We adopt Bourbaki's numbering of simple roots; see [Bourbaki 2002]. Since $\chi \neq 0$ and the adjoint $G$-orbit of $e_{\alpha_{7}}$ spans $\mathfrak{g}$ by the simplicity of $\mathfrak{g}$, we may assume that $\chi\left(e_{\alpha_{7}}\right) \neq 0$. If $\chi\left(h_{\alpha_{6}}\right) \neq 0$ and $\chi\left(h_{\alpha_{8}}\right) \neq 0$, then we can take $\mathfrak{s}_{1}=F e_{\alpha_{6}} \oplus F h_{\alpha_{6}} \oplus F e_{-\alpha_{6}}$ and $\mathfrak{s}_{2}=F e_{\alpha_{8}} \oplus F h_{\alpha_{8}} \oplus F e_{-\alpha_{8}}$. If this is not the case, then we replace $\mathfrak{t}$ by $\left(\operatorname{Ad} x_{\alpha_{7}}\left(t_{0}\right)\right)(\mathfrak{t})$ for a suitable $x_{\alpha_{7}}\left(t_{0}\right)$ in the unipotent root subgroup $U_{\alpha_{7}}$ of $G$.

There exists $b \in F^{\times}$such that for every $t \in F$ we have

$$
\left(\operatorname{Ad} x_{\alpha_{7}}(t)\right)\left(h_{\alpha_{i}}\right)=h_{\alpha_{i}}+t b\left[e_{\alpha_{7}}, h_{\alpha_{i}}\right]=h_{\alpha_{i}}+t b e_{\alpha_{7}}, \quad i=6,8 .
$$

As $\chi\left(e_{\alpha_{7}}\right) \neq 0$, we can find $t_{0} \in F$ such that

$$
\chi\left(h_{\alpha_{6}}+t_{0} b e_{\alpha_{7}}\right) \neq 0 \text { and } \chi\left(h_{\alpha_{8}}+t_{0} b e_{\alpha_{7}}\right) \neq 0 .
$$

Hence we can take $\left(\operatorname{Ad} x_{\alpha_{7}}\left(t_{0}\right)\right)\left(\mathfrak{s}_{i}\right), i=1,2$, as our $\mathfrak{s l}_{2}$-triples. This completes the proof.

\section{References}

[Bardsley and Richardson 1985] P. Bardsley and R. W. Richardson, "Étale slices for algebraic transformation groups in characteristic p", Proc. London Math. Soc. (3) 51:2 (1985), 295-317. MR 86m:14034 Zbl 0604.14037

[Block 1962] R. Block, “Trace forms on Lie algebras”, Canad. J. Math. 14 (1962), 553-564. MR 25 \#3973 Zbl 0111.03903 
[Block and Zassenhaus 1964] R. E. Block and H. Zassenhaus, "The Lie algebras with a nondegenerate trace form”, Illinois J. Math. 8 (1964), 543-549. MR 29 \#4776 Zbl 0131.27102

[Bourbaki 2002] N. Bourbaki, Lie groups and Lie algebras. Chapters 4-6, Springer, Berlin, 2002. MR 2003a:17001 Zbl 0983.17001

[Carter 1985] R. W. Carter, Finite groups of Lie type. Conjugacy classes and complex characters, Wiley, New York, 1985. MR 87d:20060 Zbl 0567.20023

[Curtis 1960] C. W. Curtis, "Representations of Lie algebras of classical type with applications to linear groups", J. Math. Mech. 9 (1960), 307-326. MR 22 \#1634 Zbl 0089.25302

[Demazure and Gabriel 1970] M. Demazure and P. Gabriel, Groupes algébriques. Tome I: Géométrie algébrique, généralités, groupes commutatifs, Masson \& Cie, Éditeur, Paris, 1970. Avec un appendice 1t Corps de classes local par Michiel Hazewinkel. MR 46 \#1800 Zbl 0203.23401

[Dynkin 1952] E. B. Dynkin, "Semisimple subalgebras of semisimple Lie algebras", Mat. Sbornik N.S. 30(72) (1952), 349-462 (3 plates). MR 13,904c Zbl 0048.01701

[Garibaldi 2008] S. Garibaldi, "Orthogonal representations of twisted forms of SL2", Represent. Theory 12 (2008), 435-446. MR MR2465801 Zbl pre05526477

[Gross and Nebe 2004] B. H. Gross and G. Nebe, "Globally maximal arithmetic groups", J. Algebra 272:2 (2004), 625-642. MR 2005b:20091 Zbl 1113.20040

[Hiss 1984] G. Hiss, "Die adjungierten Darstellungen der Chevalley-Gruppen", Arch. Math. (Basel) 42:5 (1984), 408-416. MR 85k:20134 Zbl 0532.20022

[Hogeweij 1982] G. M. D. Hogeweij, “Almost-classical Lie algebras. I, II”, Nederl. Akad. Wetensch. Indag. Math. 44:4 (1982), 441-452, 453-460. MR 84f:17007 Zbl 0512.17003

[Humphreys 1995] J. E. Humphreys, Conjugacy classes in semisimple algebraic groups, Mathematical Surveys and Monographs 43, American Mathematical Society, Providence, RI, 1995. MR 97i:20057 Zbl 0834.20048

[Jantzen 2003] J. C. Jantzen, Representations of algebraic groups, 2nd ed., Mathematical Surveys and Monographs 107, American Mathematical Society, Providence, RI, 2003. MR 2004h:20061 Zbl 1034.20041

[Jantzen 2004] J. C. Jantzen, "Nilpotent orbits in representation theory", pp. 1-211 in Lie theory, edited by J.-P. Anker and B. Orsted, Progr. Math. 228, Birkhäuser, Boston, 2004. MR 2005c:14055 Zbl 02160654

[Laszlo and Sorger 1997] Y. Laszlo and C. Sorger, "The line bundles on the moduli of parabolic G-bundles over curves and their sections", Ann. Sci. École Norm. Sup. (4) 30:4 (1997), 499-525. MR 98f:14007 Zbl 0918.14004

[Mathieu 2000] O. Mathieu, "Classification des algèbres de Lie simples", pp. 245-286 in Séminaire Bourbaki, 1998/99, Astérisque 266, Soc. Math. de France, Paris, 2000. MR 2001h:17027 Zbl 0990.17008

[McKay et al. 1990] W. G. McKay, J. Patera, and D. W. Rand, Tables of representations of simple Lie algebras, I: Exceptional simple Lie algebras, Université de Montréal Centre de Recherches Mathématiques, Montreal, 1990. MR 92c:17015 Zbl 0728.17005

[Merkurjev 2003] A. Merkurjev, "Rost invariants of simply connected algebraic groups", pp. 101158 in Cohomological invariants in Galois cohomology, Univ. Lecture Ser. 28, Amer. Math. Soc., Providence, RI, 2003. MR MR1999385

[Premet 1995] A. Premet, "Irreducible representations of Lie algebras of reductive groups and the Kac-Weisfeiler conjecture”, Invent. Math. 121:1 (1995), 79-117. MR 96g:17007 Zbl 0828.17008 
[Richardson 1967] R. W. Richardson, Jr., "Conjugacy classes in Lie algebras and algebraic groups", Ann. of Math. (2) 86 (1967), 1-15. MR 36 \#173 Zbl 0153.04501

[Seligman 1967] G. B. Seligman, Modular Lie algebras, Ergebnisse der Math. 40, Springer, 1967. MR 0245627 Zbl 0189.03201

[Springer and Steinberg 1970] T. A. Springer and R. Steinberg, "Conjugacy classes", pp. 167-266 in Seminar on algebraic groups and related finite groups (Princeton, NJ, 1968/69), Lecture Notes in Mathematics 131, Springer, Berlin, 1970. MR 42 \#3091 Zbl 0249.20024

[Steinberg 1961] R. Steinberg, "Automorphisms of classical Lie algebras", Pacific J. Math. 11 (1961), 1119-1129. Reprinted as pp. 101-111 in his Collected Papers, American Math. Soc., Providence, 1997. MR 26 \#1395 Zbl 0104.02905

[Steinberg 1962] R. Steinberg, "Générateurs, relations et revêtements de groupes algébriques", pp. 113-127 in Colloque sur la Théorie des Groupes Algébriques (Bruxelles), Librairie Universitaire, Louvain, 1962. Reprinted as pp. 113-127 in his Collected Papers, American Math. Soc., Providence, 1997. MR MR0153677 (27 \#3638) Zbl 0272.20036

[Steinberg 1963] R. Steinberg, "Representations of algebraic groups", Nagoya Math. J. 22 (1963), 33-56. Reprinted as pp. 149-172 in his Collected Papers, American Math. Soc., Providence, 1997. MR 27 \#5870 Zbl 0271.20019

[Steinberg 1968] R. Steinberg, Lectures on Chevalley groups, Yale University, New Haven, 1968. MR 57 \#6215 Zbl 0307.22001

[Steinberg 1975] R. Steinberg, "Torsion in reductive groups", Advances in Math. 15 (1975), 63-92. Reprinted as pp. 415-444 in his Collected Papers, American Math. Soc., Providence, 1997. MR 50 \#7369 Zbl 0312.20026

[Strade 2004] H. Strade, Simple Lie algebras over fields of positive characteristic, I: Structure theory, Expositions in Mathematics 38, de Gruyter, Berlin, 2004. MR 2005c:17025 Zbl 1074.17005

[Tits 1966] J. Tits, "Classification of algebraic semisimple groups", pp. 33-62 in Algebraic groups and discontinuous subgroups (Boulder, CO, 1965), Amer. Math. Soc., Providence, R.I., 1966, 1966. MR 37 \#309 Zbl 0238.20052

Communicated by Georgia Benkart

Received 2008-07-16 Revised 2009-03-05 Accepted 2009-04-06

skip@member.ams.org

sashap@maths.man.ac.uk
Department of Mathematics and Computer Science, Emory University, Atlanta, GA 30322, United States http://www. mathcs.emory.edu/ skip/

School of Mathematics, The University of Manchester, Oxford Rd., Manchester, M13 9PL, United Kingdom 